\title{
Distributed digital real-time control system for the TCV tokamak and its applications
}

\author{
H. Anand ${ }^{1}$, C. Galperti ${ }^{1}$, S. Coda ${ }^{1}$, B.P. Duval ${ }^{1}$, F. Felici ${ }^{2}$, T. Blanken ${ }^{2}$, E. Maljaars ${ }^{2}$, J.- \\ M. Moret ${ }^{1}$, O. Sauter ${ }^{1}$, T. P. Goodman ${ }^{1}$, D. Kim ${ }^{3}$
}

${ }^{1}$ Ecole Polytechnique Fédérale de Lausanne (EPFL), SPC, CH-1015 Lausanne, Switzerland ${ }^{2}$ Eindhoven University of Technology, Mechanical Engineering, Control Systems Technology. P.O. Box 513, 5600 MB Eindhoven, The Netherlands

${ }^{3}$ ITER Organisation, Route de Vinon-sur-Verdon, CS 90 046, 13067 St.-Paul-lez-Durance Cedex, France

E-mail: himank.anand@epfl.ch

\begin{abstract}
A key feature of the new digital plasma control system installed on the TCV (Tokamak à Configuration Variable) tokamak is its possibility to rapidly design, test and deploy real-time algorithms. It accommodates hundreds of diagnostic inputs and actuator outputs, and offers the possibility to design advanced control algorithms with better knowledge of the plasma state and to coherently control all TCV actuators, including poloidal field coils, gas valves, the gyrotron powers and launcher angles of the electron cyclotron heating and current drive system together with diagnostic triggering signals. It encompasses plasma control applications ranging from basic experiments of coil current and density control to advanced experiments of magnetohydrodynamics (MHD) and plasma profile control. The system consists of multiple nodes, each of which may have a local analog to digital (ADC) and/or digital to analog (DAC) card; all nodes are connected to a reflective memory (RFM), providing a deterministic method of sharing memory between them. Recently, a generalized plasma position and shape controller based on the real-time (RT) Grad-Shafranov solver RTLIUQE was developed and implemented, providing the basis for future high performance plasma operation with advanced plasma configurations. The controller design is based on an isoflux control scheme and utilizes singular value decomposition (SVD), to respect the limits on poloidal field coils currents by limiting the controlled parameters to the set that can be more easily controlled. The controller is capable in principle of providing improved equilibrium control especially for unconventional plasma scenarios, for e.g. reliable control of 'snowflake' equilibria with closely spaced x-points, i.e. the 'exact' snowflake, and the development of negative triangularity plasmas in H-mode. An addition of a new node on the digital control system has enhanced the real time computational capacity and hosts the real-time transport code RAPTOR (rapid plasma transport simulator), an advanced density profile reconstruction algorithm including real-time fringe jump correction, as well as a plasma state monitoring, supervision and actuator management algorithm. In future, more signals from existing TCV diagnostics, including multiview pinhole Xray diagnostics, Thomson scattering, visible image processing and magnetic signals for MHD mode analysis will be added to expand the capabilities of the digital control system.
\end{abstract}

\section{Introduction}

In a tokamak reactor, real-time control is a necessity for controlling high-performance plasma discharges. Using diagnostic signals, the control system executes feedback algorithms to control plasma current, position, shape and density to sustain a pre-defined plasma equilibrium. Recently, attention has turned to advanced control schemes such as the stabilization of MHD mode instabilities [1] [2] [3] [4] and the tailoring of magnetic and kinetic profiles [5] [6] [7]. These are made possible by the real-time processing capabilities of present-day digital computational platforms. In 2008, it was decided to build a new digital system for TCV [8], featuring massive multichannel capabilities and high flexibility, allowing many more real-time diagnostic signals as inputs for real-time control, and capable of controlling all the available actuators. These efforts resulted in the new system named from the French acronym for SCD "Système de Contrôle Distribué" [9]. 
The performance of the legacy control system of TCV has been successfully replicated in the digital control system with the addition of new features for e.g. improved control of the ohmic transformer coil during non-inductive plasmas and improved density control by gain scheduling, which could not be handled by the analog electronics of the original system. Furthermore, the system has been successfully extended to advanced experiments on MHD and plasma profile control, as well as to incorporate real time plasma transport simulation. One achievement is the deployment of the RT version of the Grad-Shafranov equilibrium reconstruction code LIUQE [10], with a sub-ms cycle time. This has been employed in particular in neoclassical tearing mode (NTM) control by electron cyclotron resonance heating (ECRH) building on past experience but now benefiting from knowledge of the location of the rational q surfaces [11]. Another recent valuable application of the RTLIUQE [12] is the development of a new real-time generalized plasma position and shape controller for advanced plasma configurations [13]. A new real-time advanced magnetic analysis code has also been integrated on a newly installed real-time node of the SCD [14], which detects and analyzes plasma instabilities and helps to formulate decision making algorithms that can initiate actuator reactions to mitigate them.

The paper is structured as follows. Section 1 presents a brief overview of the digital control system, its architecture and programming methods. Section 2 provides an overview of the integration of the new real-time generalized plasma shape and position controller with the TCV hybrid controller on SCD and its experimental application to a tokamak plasma discharge. Section 3 discusses the integration of a new real-time magnetic coil analysis node on SCD and presents system tests during TCV operation. Finally, Section 4 discusses future plans for further use of the system's potential.

\section{The Distributed digital control system on TCV tokamak}

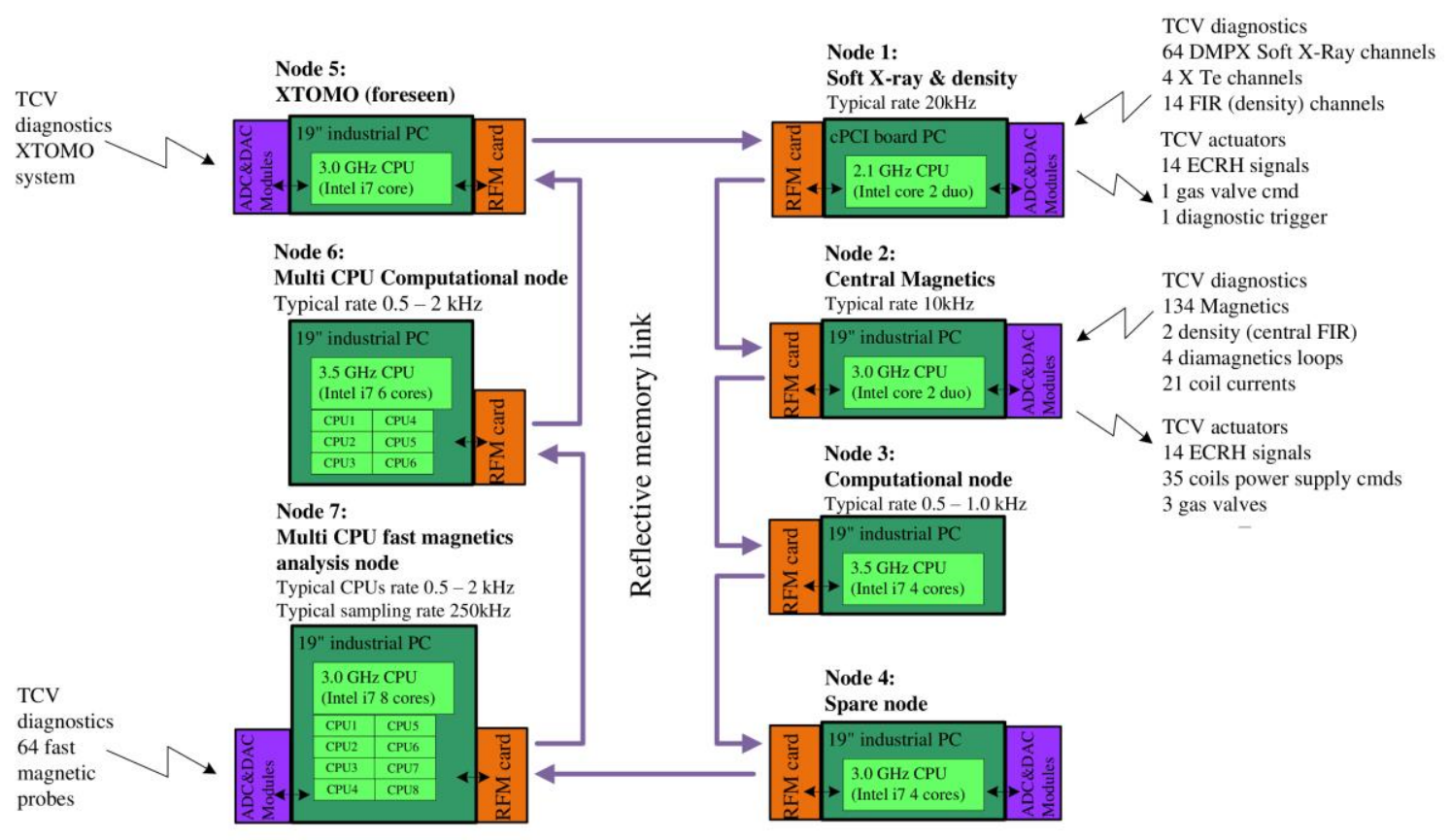

Fig. 1. Top view of the SCD

The SCD hosts diagnostic inputs and actuator outputs and consists of a set of independent nodes linked via shared reflective memory. Each node can have a different cycle time, varying between 50us and $1 \mathrm{~ms}$, depending on the needs for acquisition and computational complexity 
of algorithms. The design of the diagnostic signal processing and control algorithms is performed in Matlab-Simulink, providing a natural framework for modelling and control design. For real-time execution, $\mathrm{C}$ code is generated from the Simulink block diagram, compiled, with the Simulink Embedded Coder, into a Linux shared library and distributed to target nodes in the discharge preparation phase. During the TCV discharge, an application on each node is executed that dynamically loads the shared library at runtime. Upon the completion of the discharge, all data stored in RT nodes is copied to the host computer and archived to the TCV database.

Figure 1 shows the SCD control system layout with the connectivity to the diagnostics and actuators. The nodes with ADCs and DACs are interfaced to the machine's diagnostics systems and actuators. Node 1 is interfaced to 2 soft-X ray diagnostics (DMPX, or Duplex Multiwire Proportional soft X-ray counter, a pinhole type soft-X camera and X-Te, a four filter soft-X spectrometer that provides the central electron temperature using the differential filter method). It is also interfaced to the 14 vertical chords of the Far InfraRed (or FIR) interferometer providing the electron density profile information. Node 2 is the central core of the system and acquires all magnetic measurements from the tokamak and is thus responsible for plasma shape and position control; it also acquires the central FIR channel for real-time control of the density. This node is used routinely as the main plasma position and density controller and is almost always the RFM master node. Node 3 is a computational node that computes the plasma magnetic equilibrium in real-time. Node 4 is a hardware and software clone of node 2, It serves as a replacement node in case of node 2 failure. Node 5 is an acquisition and processing node, presently under commissioning, connected to the 200 channel soft $\mathrm{x}$-ray tomographic (XTOMO) system. Node 6 is a very recently installed multicore computational node that has been used to run multicore complex control codes (a faster real time equilibrium reconstruction replica and real-time modeling based advanced plasma performances controllers). Finally, node 7 is devoted to real-time analysis of fast magnetic perturbations in the plasma.

The link between all RT nodes is provided by reflective memory which features a $128 \mathrm{MB}$ memory area that is shared across all the nodes. A fiber optic ring network links the reflective memory network cards in each node. Data written by one node to a memory address within this shared memory area will automatically appear at the same memory address within all the other after a very short delay $(0.7 \mathrm{~ms})$. The RFM is partitioned in a small control parameter section and large data area. Each node is assigned a separate section within the data area in which to write data, preventing the nodes from overwriting data areas outside their assigned write area [15].

The SCD code is divided into two main sections:

- Hardware interface code written in $\mathrm{C} / \mathrm{C}++$ language by the system developers. This provides the input/output interface to the control algorithm code. Once compiled, the executable is uploaded to real-time computer nodes, where it is considered fixed and unchanging between plasma shots.

- Control algorithm code realized in MathWorks-Simulink block programming language by the control algorithm author, using Simulink templates given by the system developers. It performs signal processing and computational actions to provide output signals consisting of new values for the actuators, the reflective memory and other signals (probe signals) used for post-shot analysis. This algorithm is in user-friendly Simulink block format and is automatically converted into target code that is a dynamically linked shared object library by Math Works Simulink Embedded Coder. 
The SCD also provides the flexibility for verifying a new or modified algorithm before implementing it on an actual plasma discharge. The SCD architecture can be activated in background on a plasma discharge controlled by the original hybrid control system (an extensive analog multichannel multiplication system digitally reconfigurable with PID control blocks). This not only facilitates the verification of algorithms running on different nodes but also estimates the algorithm execution time with respect to each node as well. Furthermore, the digital control system can also perform runs on individual nodes in a standalone fashion or on the entire control system offline, from the tokamak. All the algorithms running on individual nodes are executed with noise in the ADC inputs, which provides an estimate of the exact execution time based on the assumption that the computational complexity of the algorithm is independent of the input ADC dataset.

\section{Generalized plasma position and shape controller}

In a tokamak, the plasma is magnetically confined through electromagnetic fields generated by a set of toroidal and poloidal field coils distributed around the vacuum vessel. The fields interact with the plasma and modify its current, position and shape. In order to maximize performanceto-cost ratio, it is advantageous to produce and maintain the plasma in vertically elongated cross-sections which result in an unstable equilibrium; in particular, the elongation causes the appearance of a vertically unstable mode [16]. As a consequence, feedback control of the plasma position is mandatory. Although the plasma-facing components are designed to withstand high heat flux [17], contact with the plasma is a major concern in tokamak operation, and therefore adequate plasma-wall clearance must be guaranteed. This clearance is obtained by means of currents generated by a power supply system driven in feedback by a plasma-shape control system. While these basic forms of control have long been implemented in the legacy analog control system of TCV, a more precise control of every aspect of the plasma position and cross-sectional shape would be beneficial for exploring new configurations and their impact on plasma performance and stability. The successful implementation of RTLIUQE [12] (RT version of the Grad-Shafranov equilibrium reconstruction code LIUQE [10]) on SCD has facilitated the design of a new generalized plasma position and shape controller, based on the information of poloidal flux and magnetic field provided by the real-time Grad-Shafranov solver.

The new generalized plasma position and shape controller algorithm uses a singular value SVD approach, to limit the controlled parameters to the set that is most easily controlled, while respecting the hardware limits on the poloidal field coil currents. The designed controller is capable of providing improved equilibrium control especially for unconventional plasma scenarios, for e.g. reliable control of 'snowflake' equilibria with closely spaced X-points (i.e., the 'exact' snowflake) [18] and the development of diverted negative triangularity plasmas in $\mathrm{H}$-mode [19]. The capability of simultaneously controlling the position, shape, divertor leg, flux expansion and $\mathrm{X}$-points for various plasma configurations is expected to be instrumental to achieving high performance in future campaigns.

Figure 2 demonstrates the implementation of the controller in conjunction with the emulation of the TCV hybrid controller on SCD. The hybrid emulation on SCD has been explained in detail in [9]. The original scheme includes a proportional $(\mathrm{P})$ and proportional plus differential (PD) control on the radial and vertical position of the plasma. The generalized plasma position and shape controller is designed to replicate the proportional part of the position control only, the differential control on the vertical position is retained in the TCV hybrid controller to provide the necessary vertical stability. 


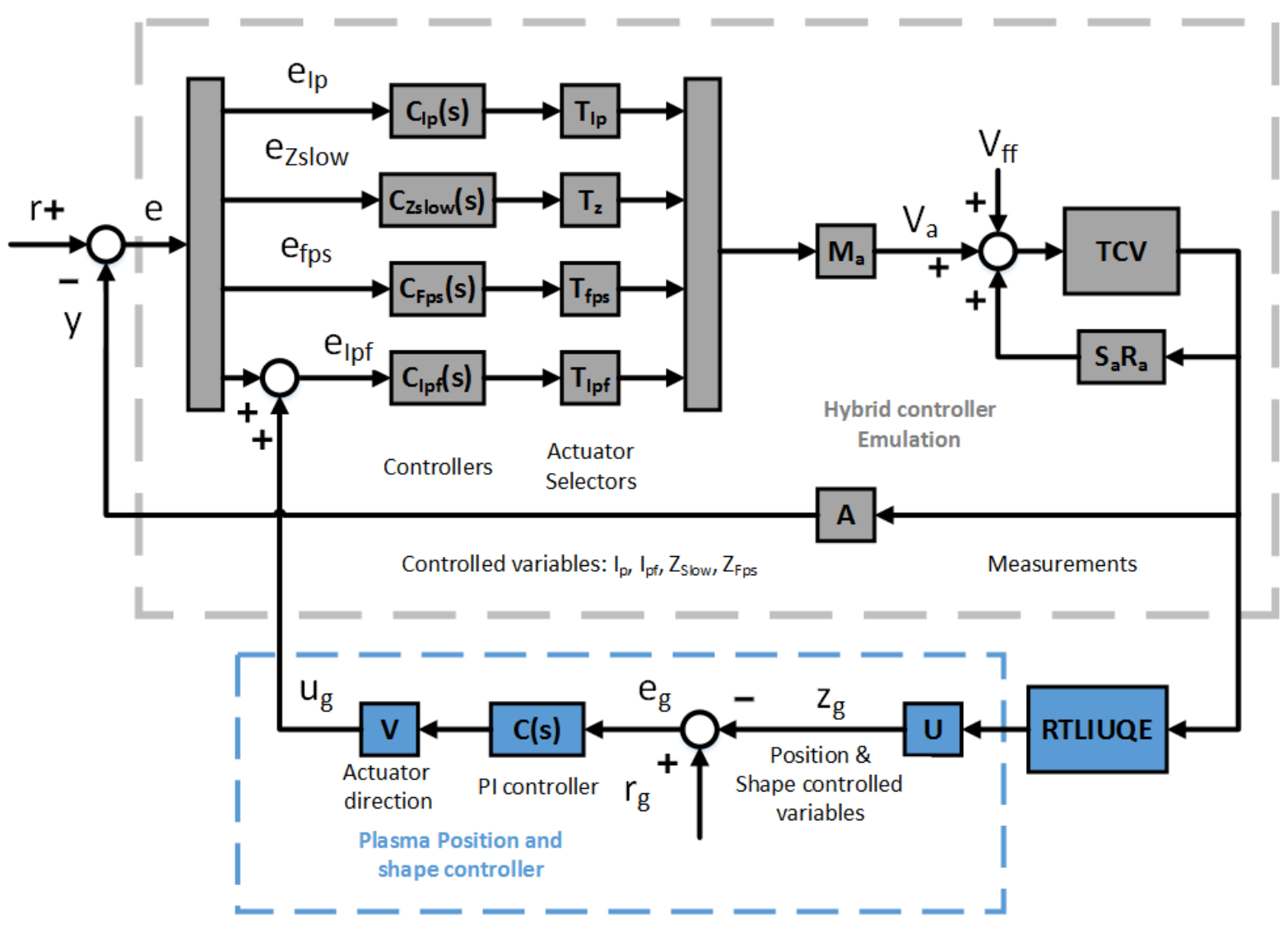

Fig. 2. Implementation of the generalized plasma position and shape controller with the TCV hybrid controller emulation on $S C D$.

A simplified scheme of the new controller is shown inside the blue box, it includes a pre multiplier matrix $U$ and a post multiplier matrix $V$ : the latter translates the RTLIUQE outputs to position and shape controlled variables, $z g$, while the former provides the optimized coil directions for controlling the plasma position and shape. $C(s)$ represents a diagonal PI controller. The grey box depicts a simplified scheme for the TCV hybrid controller (radial control loop not shown), $r$ and $y$ refer to the references and observables of the plasma current, vertical position and poloidal field coil currents, $e$ denotes the errors on the controlled variables. Matrix A, converts the measurements from the TCV tokamak into the observables. The vertical position control can be subdivided into slow and a fast control loop, the slow loop comprises of a PD ( $\left.\mathrm{C}_{\text {zslow }}(\mathrm{s})\right)$ controller while the fast loop is composed of only a differential controller $\left(\mathrm{C}_{\mathrm{Fps}}(\mathrm{s})\right)$. The slow loop actuates a combination of poloidal field coil power supplies with a slow switching time of the thyristors $(1 \mathrm{kHz})$. The vertical stabilisation of highly elongated TCV plasmas is not feasible due to the slow switching time of the poloidal field coils and the shielding effect from the vacuum vessel. Consequently, a fast power supply (FPS) is used to supply a fast internal coil with a switching frequency of $10 \mathrm{kHz} . \mathrm{C}_{\mathrm{Ipf}}(\mathrm{s})$ and $\mathrm{C}_{\mathrm{Ip}}(\mathrm{s})$ represents a simple proportional controller for controlling the plasma current and poloidal field coil currents. $\mathrm{T}_{\mathrm{Ip}}, \mathrm{T}_{\mathrm{z}}, \mathrm{T}_{\mathrm{fps}}$, and $\mathrm{T}_{\mathrm{Ipf}}$ denotes the matrix that selects the respective actuators. $\mathrm{S}_{\mathrm{a}}$ selects the poloidal field coil currents from the TCV measurements. $M_{a}$ and $R_{a}$ represents the mutual inductance and the resistance of the actuators, $V_{a}$ and $V_{f f}$ represents the feedback and the feedforward voltage of the actuators. The control structure for the TCV PID controller is described in [9]. 
A time-invariant version of the controller has been tested successfully on various TCV plasma discharges, including negative triangularity and diverted plasmas. In addition, a prototype version, capable of handling time varying shapes has also been tested successfully on both limiter and divertor plasma configurations. Figure 3 shows a successful experimental implementation of the controller on a limited tokamak plasma discharge.

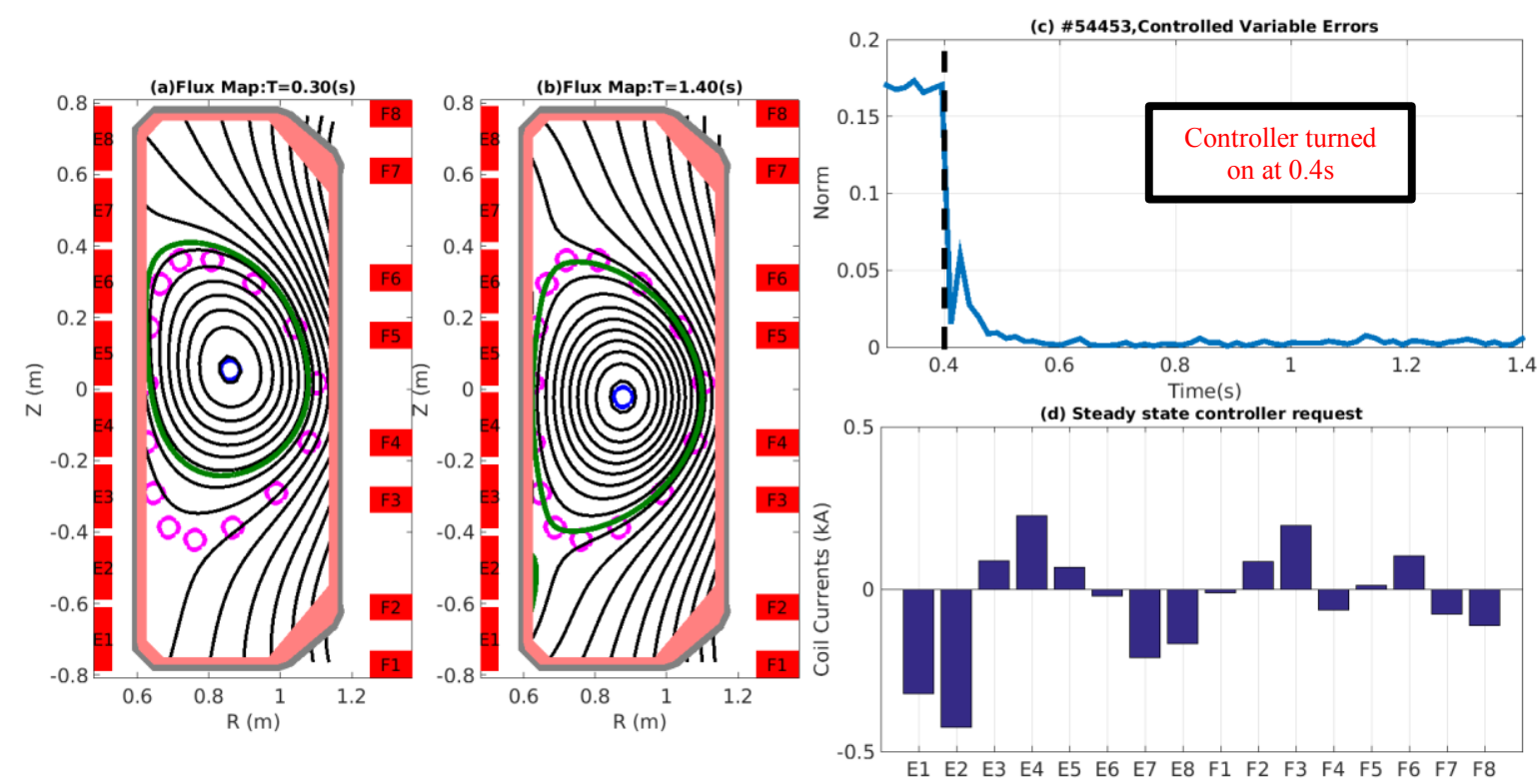

Figs. $3 a$ and $3 b$ show the poloidal flux map at time instants before and after the controller activation. The control points denote the preprogramed plasma boundary (magenta circles). E1-E8 and F1-F8 are the poloidal field coils used to control the plasma shape and position. Figs. $3 c$ and $3 d$ show the aggregate norm of errors on controlled variables as a function of time, and coil current requested by the controller at steady state.

\section{Real time magnetic analysis}

The analysis of fast magnetic signals measured by magnetic probes installed on a tokamak vessel has been widely employed to detect and analyze a number of plasma instabilities, notably rotating tearing modes (fast means faster with respect to standard magnetic signal routinely used for general plasma position and current control usually laying below few $\mathrm{kHz}$ in spectrum, the signals spans can span a range of spectrum between $5 \mathrm{kHz}$ to $50 \mathrm{kHz}$ band). The high frequency components of the measured signals contain information directly related to rapid changes within the plasma, for instance those generated by tearing mode magnetic islands rotating in the torus. Off-line analysis of these signals employs powerful mathematical tools such as spectrograms, principal component analysis and amplitude-phase fitting algorithms [20] [21] [22] [23]. These provide copious information on magnetic island topology, size and evolution. The execution of these analysis procedures during the tokamak discharge opens the way to an entire set of new experimental possibilities since a number of new plasma performance indexes are made available to the rest of the control system. Therefore, in 2015 it was decided to augment the SCD with a new processing node expressly dedicated to the realtime analysis of fast magnetic signals as the seventh node of the system.

Being devoted to real-time signal analysis, this node must comply with slightly different constraints with respect to the others nodes, in brief:

- Sampling frequency. The frequency of the usually observed plasma instabilities of the TCV tokamak lies in the range from some hundreds of $\mathrm{Hz}$ to $100 \mathrm{kHz}$. Furthermore, phase 
coherence based algorithms are often used to infer coherent plasma structures and so simultaneous sampling of signals is mandatory. Simultaneous sampling of at least 32 magnetic signals at frequencies above $200 \mathrm{ksps}$ is required.

- CPU process time. The previous requirement limits the CPU processing time since higher sampling rates lower the available processing time on the CPU (being equal to the sampling period). The aim of this node is to execute complex analysis algorithms on high speed multichannel data streams not suffering from the limitation of single sample period.

- Multi-processing capability. Processing algorithms may be spread over multiple cores to increase the computational power.

- Legacy RFM interface. Data sharing with other nodes of the control system have to comply with the existing data distribution scheme.

- Processing algorithms written in Mathworks Simulink. The analysis algorithms should be developed in Matlab/Simulink and automatically uploaded to the analysis node.

To comply with the above requirements, we adopted a packet acquisition-processing scheme where ADCs have a double buffered data path allowing the CPUs to operate on each data packet as described in [24]. The acquisition hardware employs an ADC subsystem with a D-TACQ ADC196 board augmented with a RTM-T rear transition module [25]. This module provides a high speed PCI-Express $1 \mathrm{x}$ link to the host PC, which is equipped with a motherboard hosting an Intel i7-5960X 8-core $3 \mathrm{GHz}$ processor.

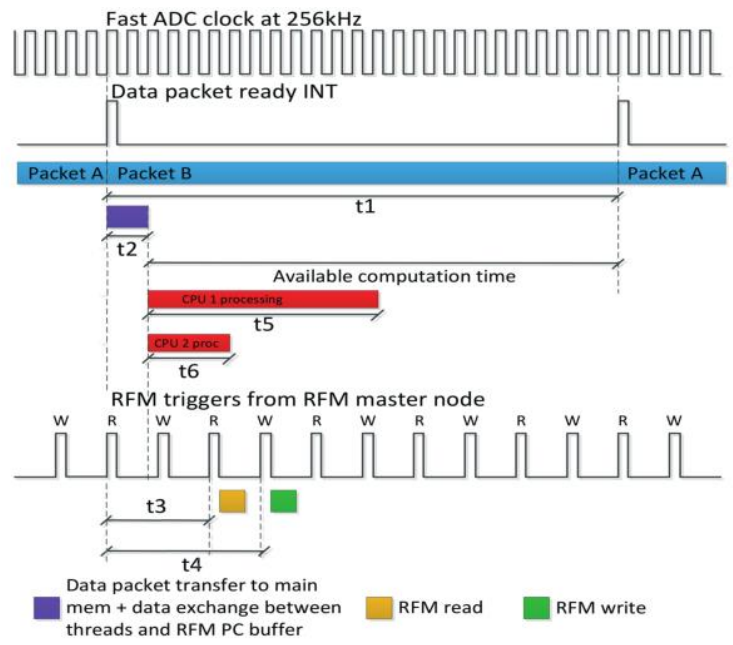

Fig. 4. Timing chart of node 7. A fast ADC clock, derived from the main TCV clock, is used to clock the ADCs. The ADC data stream is directed to a ping-pong hardware buffer located on the acquisition unit. Once a data buffer is full, it is released to the CPUs, the analysis algorithms are triggered on it and the other buffer is used to continue the acquisition. Results are distributed on the reflective memory, in sync with timings dictated by the RFM master node

The adopted kernel is Scientific Linux 6.7 especially configured for user mode RT capabilities with a dedicated hardware interface code. The industrial PC is fitted with a Reflective Memory Card of the same kind as all the other nodes of the system to communicate with real time network [26]. Figure 4 summarizes how this node works by mean of its typical timing chart.

Node 7 has been tested on real TCV plasmas during the most recent campaign with the MHD analysis code described in [27]. In essence this code employs a Singular Value Decomposition of a matrix whose columns are a band pass filtered copy of the fast magnetic signals followed by a post-processing phase that compares the experimental principal axes (i.e. the columns of one of the three matrices computed by the SV decomposition) with those computed on a 
numerically generated set of signals from a theoretical model of rotating modes. This algorithm was ported to the Simulink representation of SCD. It exploits the multiprocessing capability of node 7 operating on 2 cores: the first computes the SVD decomposition of live signals from the ADCs and the second computes the SVD decomposition of the theoretical model. Not only does this reduce the computational time but the theoretical model can be made dependent on other live plasma signals. In the presented implementation it depends upon the plasma magnetic axis in the machine. Conventional oddN and evenN MHD markers have also been included in the analysis code. Figure 5 presents the results obtained on a shot meant to have a NTM activity. The node was operated at $1 \mathrm{~ms}$ cycle time (analysis time) with the online SVD analysis computed on 12 channel sampled at $256 \mathrm{kHz}$ (so using 256 samples of data per packet). Cycle time is quite well respected as can be seen from the first plot of figure 5. The NTM markers all go off just after $\mathrm{t}=1.2$ (normalized markers SVDH and SVDP1 approach 0 and 1 respectively, normalized $(2,1)$ likelihood peaks near 0.8 and oddN markers all go high w.r.t. the baseline).
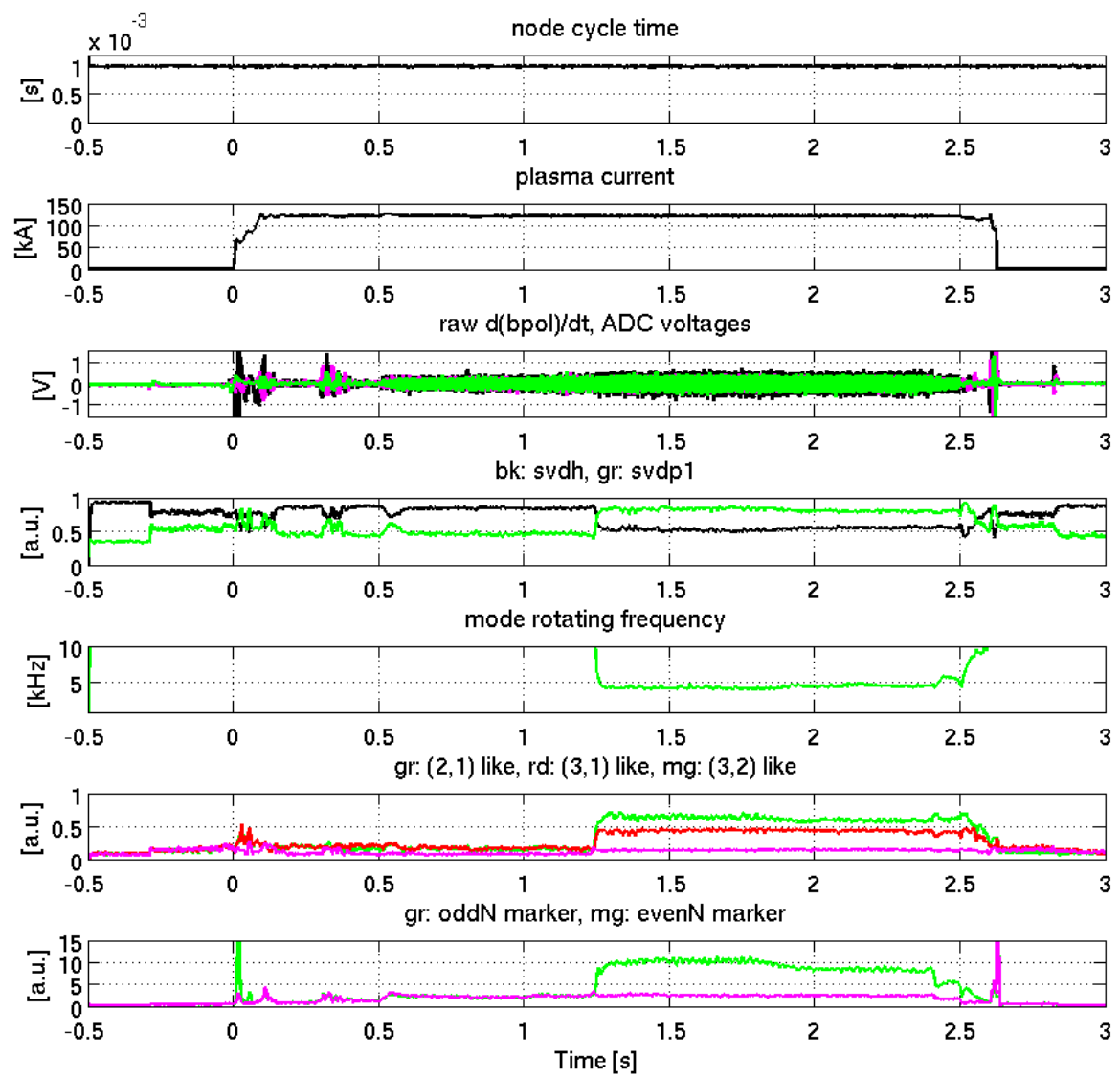

Fig. 5. Results of node 7 loaded with the SVD based MHD analysis code on a real shot. First plot: node cycle time (nominal 1ms), second plot: plasma current, third plot: fast magnetic raw signals, fourth plot: normalized NTM activity presence markers SVDH and SVDP1, fifth plot: mode rotation frequency, sixth plot: normalized $(2,1),(3,1)$ and $(3,2)$ modes likelihood, seventh plot: conventional oddN and evenN markers.

All these signals can be used to trigger a NTM control action on the $q=2$ rational surface. Finally, note that the $(2,1)$ rotating mode is barely seen from the raw signals (even looking at the amplitude variation), and only a thorough signal filtering and analysis allows one to compute the presented results. 


\section{Conclusion}

The development and installation of the distributed digital control system has provided the ability to control the entire TCV actuator set, including PF coils, gas valves, and the ECRH/ECCD system in real-time. The control algorithm design in user-friendly Simulink environment together with the automatic code generation using Simulink Embedded Code have proven to be powerful tools to rapidly design and implement control algorithms, a recent example being the generalized plasma position and shape controller. The controller has been successfully tested on various complex TCV plasma configurations. The addition of a real time magnetic analysis node to the control system offers the possibility to design advanced control algorithms using information on the plasma state to improve plasma performance and stability.

To enhance the efficiency of the digital control system, it is planned to implement the capability of simulating control algorithms on various nodes with previous shot data at the level of the ADC inputs. This will provide the feature of pre-testing the control algorithms in an environment that is very similar to the final control system. It is planned to expand the capabilities of the digital control system to add more signals from existing TCV diagnostics, including multiview pinhole X-ray diagnostics, Thomson scattering and visible image processing. For some of these applications, a new type of node has been developed which can process packets of digital signals sampled at high speed and processed in windows, allowing advanced signal processing algorithms to be applied to full bandwidth diagnostic signals up to $500 \mathrm{kHz}$. At this time, stabilization of TCV plasma vertical position using internal coils is handled by the legacy analog controller system as the latency of the digital control system is too large, but systems based on field programmable gate arrays (FPGA) are planned to be used for this task in the future.

\section{Acknowledgment}

This work has been carried out within the framework of the EUROfusion Consortium and has received funding from the Euratom research and training programme 2014-2018 under grant agreement No 633053. The views and opinions expressed herein do not necessarily reflect those of the European Commission.

The views and opinions expressed herein do not necessarily reflect those of the ITER Organization.

\section{References}

[1] Sauter, O. et al., Physics of Plasmas 4 (1997) 1654

[2] Chapman, T. et al., Physical Review Letter. 105 (2010) 255002

[3] La, J. et al., Physics of Plasmas 13 (2006) 055501

[4] Henderson, M. et al., Nuclear Fusion 48 (2008) 054013

[5] ITER Physics Basis, Ch.6, Nuclear Fusion 47 (2007) S285.

[6] Moreau, D. et al., Nuclear Fusion 51 (2011) 063009.

[7] Ou, Y. et al., Plasma Physics and Controlled Fusion 50 (2008) 115001.

[8] Hofmann, F. et al., Plasma Physics and Controlled Fusion 36 (1994) B277

[9] Paley, J.I. et al., $17^{\text {th }}$ IEEE-NPSS Real Time Conference (2010) 1

[10] Hofmann, F. et al., Nuclear Fusion 28 (1988) 1871

[11] Felici, F. et al., Nuclear Fusion 52 (2012) 074001 
[12] Moret, J. et al., Fusion Engineering and Design 91 (2015) 1

[13] Anand, H. et al., Bull. Am. Phys. Soc. 60 (2015) DPP.GP12.140

[14] Galperti, C. et al., Plasma Physics and Controlled Fusion 56 (2014) 114012

[15] Le, H.B. et al., Fusion Engineering and Design 89 (2014) 155

[16] Lazarus, E. et al., Nuclear Fusion 30 (1990) 111

[17] Bolt, H. et al., Journal of Nuclear Materials 307311 (2002) 43

[18] Piras, F. et al., Plasma Physics and Controlled Fusion 51 (2009) 5

[19] Medvedev, S. et al., Nuclear Fusion 55 (2015) 063013

[20] Nardone, C. et al., Plasma Physics and Controlled Fusion 34 (1992) 1447

[21] Dudok de Wit, T. et al., Physics of Plasmas 1 (1994) 3288

[22] Schittenheim, M. et al., Nuclear Fusion 37 (1997) 1255

[23] Kim, J.S. et al., Plasma Physics and Controlled Fusion 41 (1999) 1399

[24] Galperti, C. et al., Fusion Engineering Design 89 (2014) 214

[25] http://www.d-tacq.com/acq196cpci.shtml

[26] http://www.ge-ip.com/reflectivememorynetworks

[27] Galperti, C. et al., Plasma Physics and Controlled Fusion 56 (2014) 114012 